\title{
Sensibilidad postoperatoria tras el cementado en prótesis fija
}

\author{
Del Castillo Salmerón R*, Maroto García J*, Ruiz Miranda M**
}

\section{RESUMEN}

De entre las complicaciones postoperatorias en prótesis fija, la sensibilidad ocupa un lugar destacado para algunos autores. Se evaluó la frecuencia de sensibilidad postoperatoria en dos grupos: (1) cementando una prótesis fija sólo con ionómero de vidrio -IV- y (2) añadiendo previamente al cementado con IV un agente desensibilizante. Se puede concluir que la aplicación de un desensibilizante no supuso una mejoría clínica significativa en la sensibilidad postoperatoria.

Palabras clave: Sensibilidad postoperatoria, prótesis fija, cementado.

\begin{abstract}
Between the most frequent complications in partial fixed prosthodontics, the postoperative sensitibity occupies an outstanding place for several authors. In this work was evaluated the frequency of postoperative sensitibity in 42 patients with full-coverage restorations were cemented with glass ionomer (group 1) or dentin bonding agent and glass ionomer (group 2). There were not differences between the groups.
\end{abstract}

Key words: Postoperative sensitibity, partial fixed prosthodontic, cementation.

* Profesor Titular. Facultad de Odontología. Universidad de Granada.

* Profesor Asociado. Facultad de Odontología. Universidad de Granada.

Ramón del Castillo Salmerón, José Maroto García, Miguel Ruiz Miranda. Sensibilidad postoperatoria tras el cementado en prótesis fija. Av. Odontoestomatol 2004; 20-5: 233-237.

\section{INTRODUCCIÓN}

El éxito clínico de una prótesis fija está íntimamente relacionado con el material de cementado y la técnica de cementación (Diaz Arnold y cols, 1999). Las complicaciones postoperatorias en prótesis fija son relativamente frecuentes. De entre las complicaciones postoperatorias, la sensibilidad ocupa un lugar destacado para algunos autores, estimándola en un 10-35\% (Rosenstiel S F y cols, 1998). En cambio, otros lo consideran un problema insignificante (Pameijer CH, 1994). Un acercamiento empírico al problema nos demuestra que la sensibilidad postoperatoria es una realidad clínica nada insignificante y que puede afectar de forma importante a la calidad de vida oral del paciente. Entre las causas de sensibilidad postoperatoria se han citado las siguientes:

- Tallado excesivo.

- Sobrecalentamiento (durante tallado, confección de provisionales).

- Tipo de rotatorio.

- Contaminación microbiana.

- Tipo de cemento.

- Desecación.

Uno de los sistemas propuestos para evitar la sensibilidad postoperatoria ha sido la aplicación de un agente desensibilizante sobre los muñones tallados. 
En este trabajo nos planteamos los siguientes Objetivos:

1) Conocer la frecuencia con la que se produce sensibilidad postoperatoria en la muestra.

2) Conocer si el uso de un desensibilizante afectaba a la frecuencia de aparición de sensibilidad postoperatoria.

\section{PACIENTES Y MÉTODOS}

Un total de 42 pacientes tratados con una Prótesis Fija de tres, cuatro o cinco piezas, Fueron distribuidos alternativamente en 2 grupos de 21 pacientes cada uno. Los criterios de inclusión fueron:

- Ambos pilares fueron siempre dientes vitales.

- No sensibilidad preoperatoria en el cuadrante.

- Talladas por un único operador.

\section{- Grupo 1: CEMENTADO CON IONOMERO DE} VIDRIO SOLO.

\section{- Grupo 2: APLICACIÓN DE UN DESENSIBILIZAN-} TE Y CEMENTADO CON IONOMERO DE VIDRIO.

\section{Grupo 1}

Tras el tallado se procedió a la realización de coronas provisionales que fueron cementadas con Temp Bond $^{\circledast}$ y se aguardó una semana para la toma de impresiones.

La toma de impresión se realizó con Optosil ${ }^{\mathbb{}} \mathrm{y}$ Xantopren mediante una técnica de doble impresión. Se tomaron registros para el montaje en articulador. Tras ella se volvieron a cementar las coronas provisionales. • Se realizó prueba de estructura metálica.

El cementado se realizó con ketac-cem ${ }^{\circledast}$ (Espe-premier) siguiendo las especificaciones del fabricante para su mezcla. Se limpiaron los pilares de restos de cemento provisional con piedra pómez húmeda y copa de goma. se lava y seca. Se aislaron con rollos de algodón y eyector de saliva. Se mezcla el cemen- to y se coloca con un pincel en la corona llevándose a continuación a boca y asentándose completamente. - Se mantiene el área de trabajo seca hasta que endurece y se aplica una capa de vaselina para evitar que se deshidrate.

- Una vez completado el fraguado se retiran los excesos y se vuelve a aplicar vaselina sobre los márgenes.

-Finalmente se chequea la oclusión.

\section{Grupo 2}

En el grupo dos se realizó una aplicación de desensibilizante (Quadrant-FiniSense ${ }^{\circledR}$ Cavex) a la terminación del tallado.

- Secado del muñon.

- Aplicación del desensibilizante 30 seg.

- Secado del muñon.

- Lavar con agua

- Los siguientes pasos fueron los mismos.

\section{CONDICIONES DEL ENSAYO}

El tiempo desde la toma de impresión hasta la cementación fue estandarizado a 2 semanas. La lectura de la sensibilidad postoperatoria fue realizada por otro operador que no conocía la asignación de los pacientes a cada grupo. Los pacientes tan poco conocían a que grupo fueron asignados. El Método de determinación de la sensibilidad postoperatoria fue el siguiente:

\section{ESCALA ORDINAL:}

- $0=$ No sensibilidad

- 1 = Tiene sensibilidad pero puede masticar por ese lado.

- 2 = Tiene sensibilidad y evita masticar por ese lado,

- 3= Precisa tratamiento.La determinación de la sensibilidad postoperatoria fue realizada:

- En la primera semana siguiente a la cementación.

- Al mes.

- A los seis meses. 


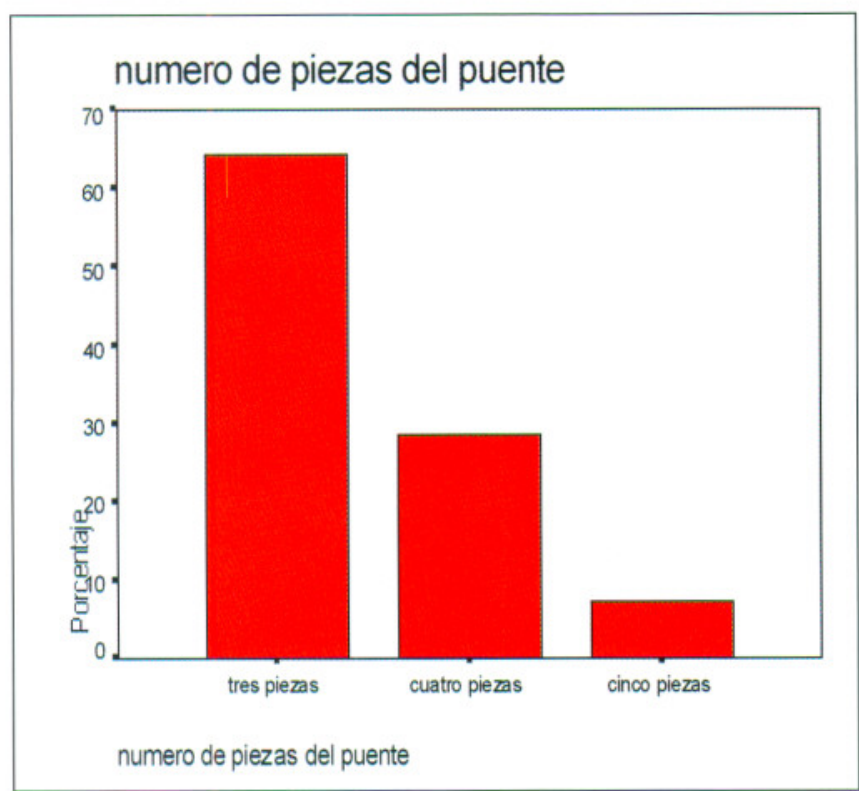

Fig. 1

- Al año.variables recogidas fueron las siguientes: Edad, sexo, número de piezas de la prótesis, tipo de cementado, sensibilidad inmediata, sensibilidad al mes, sensibilidad a los 6 meses, sensibilidad al año.

El método estadístico se realizó mediante Estadística descriptiva, Estadística inferencial: prueba de chi quadrado, realizadas mediante SPSS 9.0 para windows.

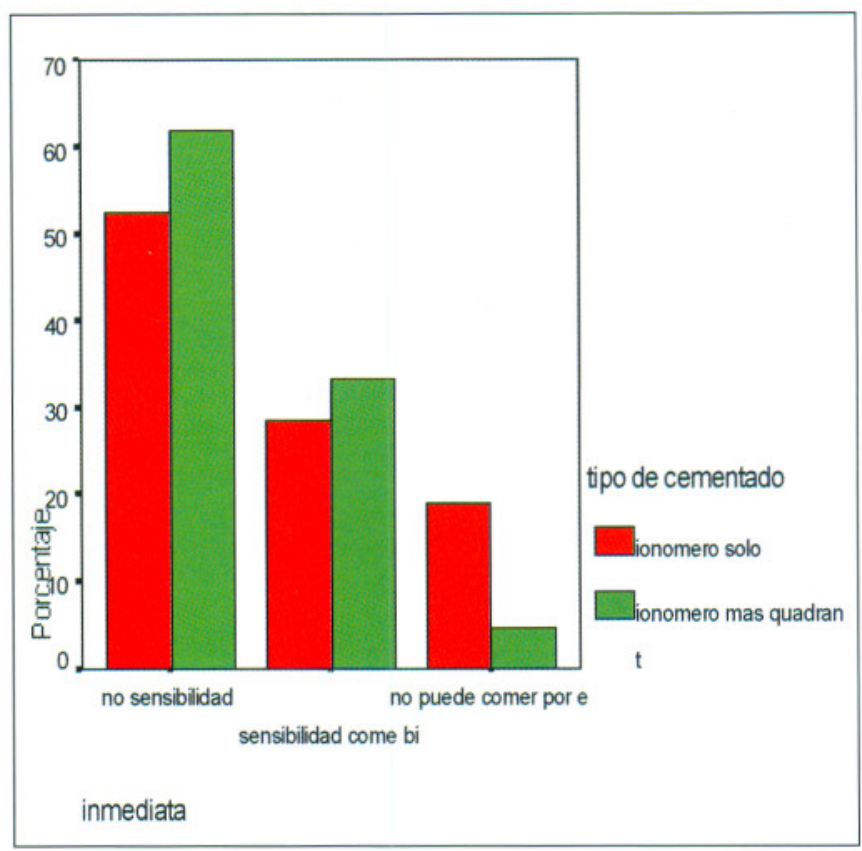

Fig. 2

La edad media fue de 36.33 con un rango entre 22 y 55 años.

- La distribución por sexos fue:

- Mujeres= 29 (69\%)

- Hombres= 13 (31\%)

- Distribución del numero de piezas del puente en el conjunto de la muestra (figura 1).

\begin{tabular}{|c|c|c|c|c|}
\hline \multicolumn{5}{|c|}{ TABLA 1.- INMEDIATA } \\
\hline & Frecuencia & Porcentaje & $\begin{array}{c}\text { Porcentaje } \\
\text { válido }\end{array}$ & $\begin{array}{c}\text { Porcentaje } \\
\text { acumulado }\end{array}$ \\
\hline Válidos No sensibilidad & 24 & 57,1 & 57,1 & 57,1 \\
Sensibilidad come bien & 13 & 31,0 & 31,0 & 88,1 \\
No puede comer por el lado & 5 & 11,9 & 11,9 & 100,0 \\
Total & 42 & 100,0 & 100,0 & \\
\hline
\end{tabular}

\begin{tabular}{|l|c|c|c|c|}
\hline \multicolumn{5}{|c|}{ TABLA 2.- 1 MES } \\
\hline & Frecuencia & Porcentaje & $\begin{array}{c}\text { Porcentaje } \\
\text { válido }\end{array}$ & $\begin{array}{c}\text { Porcentaje } \\
\text { acumulado }\end{array}$ \\
\hline Válidos No sensibilidad & 27 & 64,3 & 64,3 & 64,3 \\
Sensibilidad come bien & 8 & 19,0 & 19,0 & 83,3 \\
No puede comer por el lado & 5 & 11,9 & 11,9 & 95,2 \\
Precisa tratamiento & 2 & 4,8 & 4,8 & 100,0 \\
Total & 42 & 100,0 & 100,0 & \\
\hline
\end{tabular}




\begin{tabular}{|c|c|c|c|c|}
\hline \multicolumn{5}{|c|}{ TABLA 3.- SEIS MESES } \\
\hline & Frecuencia & Porcentaje & $\begin{array}{c}\text { Porcentaje } \\
\text { válido }\end{array}$ & $\begin{array}{c}\text { Porcentaje } \\
\text { acumulado }\end{array}$ \\
\hline Válidos No sensibilidad & 28 & 66,7 & 66,7 & 66,7 \\
Sensibilidad come bien & 6 & 14,3 & 14,3 & 81,0 \\
No puede comer por el lado & 2 & 4,8 & 4,8 & 85,7 \\
Precisa tratamiento & 6 & 14,3 & 14,3 & 100,0 \\
Total & 42 & 100,0 & 100,0 & \\
\hline
\end{tabular}

\begin{tabular}{|c|c|c|c|c|}
\hline \multicolumn{5}{|c|}{ TABLA 4.- AL AÑO } \\
\hline & Frecuencia & Porcentaje & $\begin{array}{c}\text { Porcentaje } \\
\text { válido }\end{array}$ & $\begin{array}{c}\text { Porcentaje } \\
\text { acumulado }\end{array}$ \\
\hline Válidos No sensibilidad & 28 & 66,7 & 66,7 & 66,7 \\
Sensibilidad come bien & 6 & 14,3 & 14,3 & 81,0 \\
No puede comer por el lado & 2 & 4,8 & 4,8 & 85,7 \\
Precisa tratamiento & 6 & 14,3 & 14,3 & 100,0 \\
Total & 42 & 100,0 & 100,0 & \\
\hline
\end{tabular}

- Sensibilidad postoperatoria inmediata en el conjunto de la muestra (tabla 1).

- Sensibilidad postoperatoria al mes en el conjunto de la muestra (tabla 2)

- Sensibilidad postoperatoria a los seis meses en el conjunto de la muestra (tabla 3)

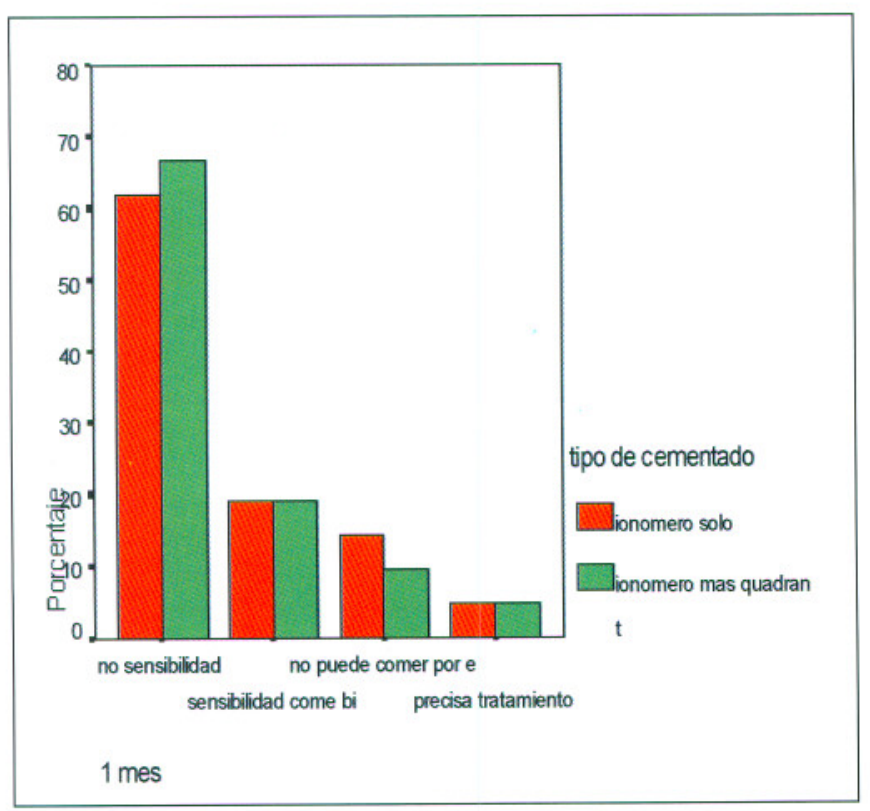

Fig. 3
- Sensibilidad postoperatoria al año en el conjunto de la muestra (tabla 4)

- Sensibilidad postoperatoria inmediata según el tipo de cementado (figura 2) (n.s.= no significativo).

- Sensibilidad postoperatoria al mes según el tipo de cementado (figura 3).

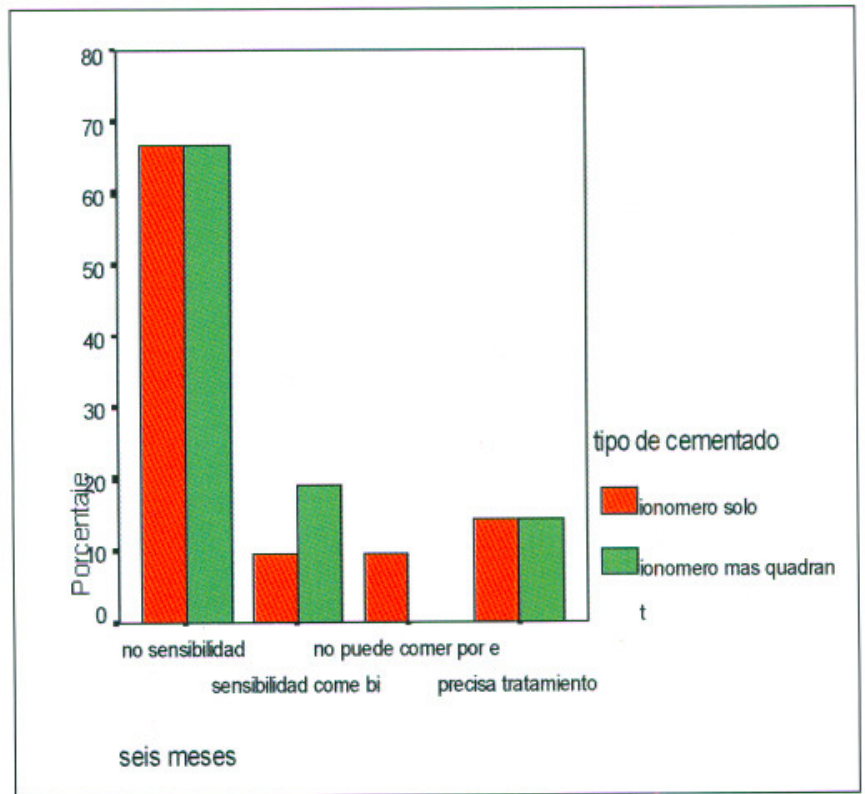

Fig. 4 


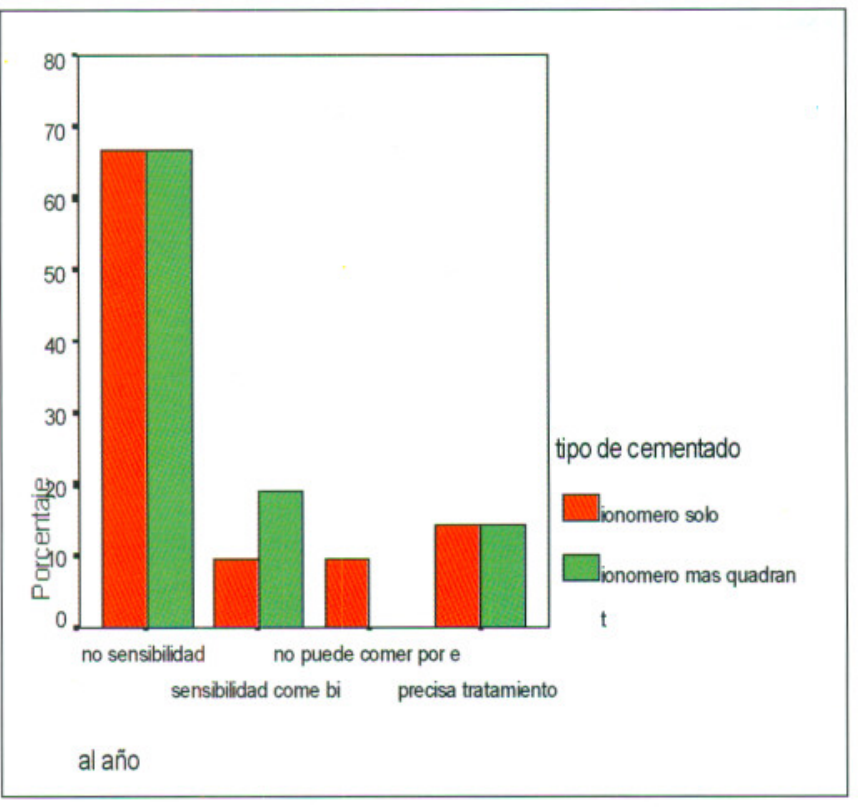

Fig. 5

- Sensibilidad postoperatoria a los 6 meses según el tipo de cementado (figura 4).

- Sensibilidad postoperatoria al año según el tipo de cementado (figura 5).

\section{DISCUSIÓN}

La sensibilidad postoperatoria ha sido encontrada con diversa frecuencia en la literatura consultada.
Rosenstiel (2000) encuentra similares a los encontrados por nosotros, aunque este autor no evalúa la evolución del cuadro.

Que duda cabe que las variables que pueden influir en la aparición de sensibilidad postoperatoria son muchas y por lo tanto el control de todas ellas es difícil por parte del investigador, pero los datos obtenidos por nosotros rechazan la apreciación de otros autores que la consideran simplemente anecdótica y no soportada por ensayos clínicos.

La atribución al cemento de ionómero de una mayor frecuencia de sensibilidad post cementación que otros cementos ha sido ,atribuida al $\mathrm{pH}$ bajo y a su sensibilidad a la manipulación, especialmente a la desecación.

\section{CONCLUSIONES}

Con las limitaciones de nuestro estudio se pueden extraer las siguientes conclusiones:

1) La frecuencia de sensibilidad postoperatoria fue alta en ambos grupos.

2) La aplicación de un desensibilizante no supuso una mejoría clínica significativa en la sensibilidad postoperatoria. 